\title{
TRIBUNAS
}

\section{Derecho, técnica y riesgo: el principio de precaución en el ámbito medioambiental}

\author{
Juan Antonio Carrillo Donaire'
}

Resumen: El factor que actualmente condiciona y determina el ejercicio de las funciones públicas de prevención y corrección del riesgo es la existencia de un presupuesto de hecho de naturaleza científica y técnica como un factor incontrolable para la Administración, cuyo dominio directo está en manos de sujetos privados especializados, de profesionales y del propio mercado.

La ordenación jurídica se vuelve más procedimental y formalista, pero también más principialista y finalista que son las notas que caracterizan a un Derecho orientado antes a los resultados que a los medios. La mayor labilidad del principio de legalidad alcanza su mayor intensidad en supuestos de riesgos graves y desconocidos por la emergencia del llamado "principio de precaución" como cláusula habilitadora de medidas excepcionales frente a los riesgos ambientales e industriales.

Palabras clave: política medioambiental, principio de precaución, riesgos del progreso.

\section{Law, technology and risk: the pre- cautionary principle in the environ- mental field}

Abstract: The factor that currently drives and determines the public functions in the area of prevention and correction of risk is the existence of an alleged event of scientific
Droit, technique et risques: le principe de précaution dans le domaine environnemental

Résumé: Le facteur qui actuellement conditionne et détermine l'exercice des fonctions publiques de prévention et de correction du risque est l'existence d'un présupposé de

1 Departamento de Derecho. Universidad Loyola Andalucía. 
and technical nature which is beyond the control of the Public Administration and whose direct ownership is under the control of specialised private parties, professionals or in the hands of the market.

The legal system becomes more procedural and formalistic but also more principlist and finalist. The latter are the issues that characterise a law more result-oriented rather than input-oriented. The greatest weakness of the principle of legality is more intense in cases of serious risks and unknown, due to the emergence of the "precautionary principle" as an enabling clause for exceptional measures against environmental and industrial risks.

Keywords: environmental policy, precautionary principle, risk of management. nature scientifique et technique incontrôlable par l'Administration, puisque son contrôle direct est entre les mains de sujets privés spécialisés, de professionnels et du propre marché.

L'encadrement juridique devient plus une question de procédure et de forme, mais aussi plus une question de principes et d'objectifs, caractéristiques d'un Droit orienté plutôt vers les résultats que vers les moyens. La plus grande labilité du principe de légalité atteint sont apogée dans l'hypothèse de risques graves et inconnus, dû à l'urgence du nommé «principe de précaution» comme clause d'habilitation de mesures exceptionnelles face à des risques environnementaux et industriels.

Mots clés: politique de l'environnement, principe de précaution, risques du progrès.

Recibido: 6 de enero de 2016 .

\section{La búsqueda jurídica de un equilibrio entre tecnocracia y riesgos del progreso}

El capítulo quinto de LS indica líneas de acción para salir de la espiral de autodestrucción en la que estamos sumergidos y contiene un punto segundo de orientación al diálogo hacia nuevas políticas nacionales y locales, en el que se hace una reflexión sobre el trasfondo de las relaciones entre el derecho, la técnica y el riesgo. Para el Santo Padre, la misión del derecho -del Derecho ambiental en particular- es propiciar un el punto de equilibrio entre el progreso tecnológico y los riesgos que el mismo conlleva ante la utilización irresponsable de las capacidades humanas en un contexto global en el que se ha desmesurado el paradigma tecnocrático y el antropocentrismo. Por ello LS afirma que los Estados tienen funciones impostergables en orden a planificar, coordinar, vigilar y sancionar, dentro de su propio territorio, el devenir de las constantes innovaciones tecnológicas y su impacto sobre el medio ambiente; de modo que el Derecho actúe de "moderador ejecutivo", 
estableciendo y haciendo cumplir "las reglas para las conductas admitidas a la luz del bien común". Concretamente, LS señala que:

Los límites que debe imponer una sociedad sana, madura y soberana se asocian con: previsión y precaución, regulaciones adecuadas, vigilancia de la aplicación de las normas, control de la corrupción, acciones de control operativo sobre los efectos emergentes no deseados de los procesos productivos, e intervención oportuna ante riesgos inciertos o potenciales (LS 177).

El factor que actualmente condiciona y determina el ejercicio de las funciones públicas de prevención y corrección del riesgo a las que se refiere este punto de LS es la existencia de un presupuesto de hecho de naturaleza científica y técnica -cada vez más denso y relevante- que se erige como un arcano inaprensible para los poderes públicos.

La complejidad técnica que preside la reacción jurídica frente el riesgo está alumbrando una nueva concepción de la intervención pública que rebasa las tradicionales formas policiales de intervención administrativa en la actividad económica y productiva sobre las actividades generadoras de riesgo, para definir un difícil equilibrio entre la permisión de las actividades con riesgo y sus límites y prohibiciones, donde la clave radica en la búsqueda de criterios para la apreciación jurídica de los conceptos imprecisos: el del riesgo tolerable y el que no lo es. En la fijación de dicho criterio, los principios jurídicos de previsión y precaución cobran un protagonismo definitorio ante el riego intolerable y la amenaza de la autodestrucción depredadora.

La insuficiencia de las clásicas medidas correctivas de la policía administrativa, de carácter puntual e imperativo, ha determinado la emergencia de soluciones jurídicas que rompen el esquema clásico en un triple plano subjetivo, funcional e instrumental.

El plano funcional muestra una evolución de la acción correctiva puntual a una acción integral que pone el acento en la dimensión preventiva. En el plano instrumental se advierte el afloramiento de técnicas jurídicas nuevas que fuerzan la reconstrucción de categorías jurídicas seculares ligadas al sistema de fuentes, a la técnica autorizatoria, el procedimiento administrativo o a la responsabilidad.

El plano subjetivo está marcado por la retirada de la Administración y la correlativa privatización de la gestión del riesgo que se traduce en el mayor protagonismo de la sociedad y del mercado, donde habita hoy el conocimiento experto. Más allá de una inicial colaboración de carácter meramente instrumental, el Derecho ha 
acabado por reconocer efectos jurídico-administrativos a las referencias técnicas generadas y a los controles ejercidos por estos sujetos especializados.

\section{El papel del derecho frente a los riesgos del progreso}

Las relaciones entre técnica y derecho se han alterado profundamente en el contexto de la llamada "sociedad del riesgo" 2 , en la que los peligros y amenazas potenciales no tienen ya su origen en una naturaleza indómita, sino en la actividad humana que persigue dominarla y ponerla a su servicio mediante el conocimiento científico y su aplicación técnica y tecnológica.

Una de las finalidades principales del desarrollo científico es paliar los peligros naturales, las amenazas y los riesgos conocidos. Pero, paradóiicamente, a medida que avanza el conocimiento científico, la industrialización y la técnica generan nuevos riesgos ("del progreso" o ligados a él) que muchas veces son imprevisibles $y$, en gran parte, desconocidos. Estos riesgos inherentes al desarrollo se manifiestan en todas y cada una de las actividades económicas y sociales. No existen áreas o parcelas libres de su impacto, que se hace sentir de modo especialmente intenso y grave en el orden medioambiental.

El concepto de riesgo empieza a tomar cuerpo allí donde se desvanece la confianza en la seguridad, y deja de ser relevante cuando ocurre el daño o la catástrofe potencial. El concepto habita, por tanto, en una zona, intermedia entre la seguridad y la consumación de un daño, en la que reina la incertidumbre, adoptando la forma de un cálculo de probabilidades que expresa las posibles consecuencias indeseables ligadas a una determinada tecnología cuya aparición o efectos no se pueden determinar a priori. Desde un punto de vista cualitativo, los riesgos del progreso se caracterizan porque uno de los dos factores del riesgo, el daño posible, es esencialmente indeterminado y en gran medida imprevisible, de pronóstico incierto

\footnotetext{
2 Término acuñado en la obra de Ulrich Beck La sociedad del riesgo; cfr. U. BECK (2002) La sociedad del riesgo. Hacia una nueva modernidad (original alemán, Francfort del Meno, 1986), Barcelona, Paidós, cuya repercusión ha sido enorme, como muestran: A. GIDDENS, Z. BAUMAN, Z. LUHMANN y U. BECK (1996) Las consecuencias perversas de la modernidad: modernidad, contingencia y riesgo, Barcelona, Anthropos; B. AdAM, U. BECK y J. VAN LOON (1999) Positioning Risk, Londres, Sage; N. LUHMAN (1993) Risk: A Sociological Theory, Nueva York, Aldine de Gruyter; o J. L. Luján LóPEZ y J. ECheVERRía (2004) Gobernar los riesgos. Ciencia y valores en la sociedad del riesgo, Madrid, Biblioteca Nueva. El propio Beck recogió parte de las críticas y sugerencias formuladas a su teoría en U. BECK (2002) La sociedad del riesgo global, Madrid, Siglo XXI.
} 
y conjetural. Desde un punto de vista cuantitativo, también es característico de este tipo de riesgos que el otro factor determinante de los mismos, la probabilidad de que el daño acontezca, se multiplica exponencialmente en una espiral creciente que es directamente proporcional al aumento del desarrollo tecnológico. Por ello, los riesgos del progreso constituyen un poderoso factor de desestabilización que cuestiona constantemente el papel y la legitimidad de las instancias políticas y administrativas con responsabilidades de control de la salud y la seguridad en materia de consumo o sobre la protección ambiental.

En la conjura de este desafío, el derecho desempeña una función capital que requiere delimitar con claridad las relaciones entre técnica y derecho y el papel de cada una de estas esferas en el tratamiento de los riesgos del progreso ${ }^{3}$.

El derecho tiene una función racionalizadora de las relaciones entre técnica y riesgo y un innegable protagonismo directivo para arbitrar los criterios y procedimientos de toma de decisiones en un entorno de riesgo e incerteza. Pero, para orientar las soluciones jurídicas, el derecho ha de tomar postura ante algunas cuestiones previas: el conocimiento, el análisis y la valoración de los riesgos del progreso. En el tratamiento jurídico del riesgo pueden diferenciarse, así, tres fases que comprometen con desigual alcance al mundo del derecho: la percepción o reconocimiento del riesgo, su valoración y su gestión.

a) La cuestión de la percepción o reconocimiento del riesgo es clave, puesto que lo que determina la acción frente al riesgo es su perceptibilidad y la información disponible sobre el mismo. El conocimientoy la definición de los concretos riesgos a los que nos enfrentamos constituyen un problema especialmente agudoy difícil. La razón es que los riesgos que genera nuestra forma de vida tan tecnificada están, tanto en un plano espacial como temporal, tendencialmente abiertos e indefinidos. Espacialmente abiertos porque el riesgo tiende a mundializarse. La lluvia ácida, el cambio climático o la adherencia tóxica y el carácter imperecedero de los compuestos químicos sintéticos son ejemplos relevantes de

\footnotetext{
${ }^{3}$ Sobre esta conexión es esencial la obra de J. ESTEVE PARDO (1999) Técnica, riesgo y Derecho. Tratamiento del riesgo tecnológico en el Derecho Ambiental, Barcelona, Ariel, que el autor ha ido enriqueciendo posteriormente en J. EsteVE PARDO (2002) Autorregulación. Génesis y Efectos, Pamplona, Aranzadi; ID. (2003) "Ciencia y Derecho ante los riesgos para la salud. Evaluación, decisión y gestión": Documentación Administrativa, 255-256, 137-149; ID. (2003) "De la policía administrativa a la gestión de riesgos": Revista Española de Derecho Administrativo, 119, 323-346; ID. (2007) "La regulación de la economía desde el Estado garante", en Actas del II Congreso de la AEPDA, Thomson-Aranzadi, pp. 79-125; e ID. (2009) El desconcierto de Leviatán. Política y derecho ante las incertidumbres de la ciencia, Madrid, Marcial Pons.
} 
esta fuerza expansiva y globalizadora del riesgo. En una dimensión temporal, el riesgo tecnológico es altamente veleidoso e imperceptible, mostrándose muchas veces al conocimiento de un modo simplemente indiciario; cuando no permanece ignoto, amenazadoramente oculto hasta eclosionar en forma de amenaza real o de daño cierto. Catástrofes ecológicas como la destrucción del ozono, el cambio climático o los daños en los sistemas inmunológicos provocados por sustancias manipuladas no se han presentado como síntomas hasta años después que empezaran a producirse de modo desapercibido. Todavía son imprevisibles o desconocidos muchos efectos de la manipulación genética, de la liberación masiva de organismos transgénicos o de otras amenazas que acaso serán síntomas en el futuro, o tras combinarse con otros factores con los que puedan formar masa crítica.

Aunque la cuestión de la percepción del riesgo atañe principalmente a las ciencias naturales y experimentales, es el orden jurídico quién ha de señalar cuáles son las referencias técnicas que determinan la inocuidad o peligrosidad de los productos y actividades riesgosas; qué tipo de conocimiento -o desconocimiento sobre las causas- se halla implicado en cada caso; $y$, a la postre, qué opiniones o hechos pueden considerarse como prueba suficiente en un contexto controvertido y probabilista. Asimismo, el derecho ha de resolver cuestiones instrumentales a ésta como el procedimiento y las garantías que han de observarse para apreciar correctamente las evidencias del riesgo. $Y$ también compete al derecho la regulación del derecho-deber de información, de singular importancia en este ámbito en el la escasez de información contrasta con la imperiosa necesidad de conocer para proteger, que ha de entrañar obligaciones específicas en la generación y el manejo de la información.

b) En segundo lugar, la valoración del riesgo alude al problema ponderativo de su asunción o rechazo. Pese a necesitar del antecedente del experto, esta actividad concierne de forma directa al orden jurídico. El derecho tiene que decidir qué riesgos se asumen y cuales se rechazan mediante la definición de las nociones jurídicas de "seguridad" y "peligro", que decantan los niveles de riesgo tolerable e intolerable. El problema se reconduce a la ponderación entre bienes jurídicos contrapuestos que se imantan a uno de los polos de las ideas-fuerza que representan, respectivamente, la libertad y la seguridad. Dicho de otro modo, la valoración del riesgo requiere la discriminación entre riesgos jurídicamente relevantes y riesgos residuales, que lleva a establecer qué riesgos conocidos o reconocibles son asumibles y cuáles no en función de los bienes jurídicos en juego o los afectados potenciales. En definitiva, esta operación consiste en calificar como riesgo la probabilidad de que ocurra un daño 
que se considera antijurídico y diseñar, mediante un sistema de presunciones, mecanismos capaces de definirlo y minimizarlo, a partir de los cuales puedan establecerse consecuencias jurídicas.

c) En tercer y último lugar, la gestión del riesgo alude a la toma de decisiones frente al mismo, que es donde se aprecia con toda su agudeza la tensión entre el elemento científico-técnico y el elemento jurídico. En ella se trata de decidir la crucial cuestión de cuáles son las medidas de protección o de reacción que deben adoptarse ante el riesgo antijurídico, junto las consiguientes garantías procedimentales. Asimismo, la gestión del riesgo ha de abordar las consecuencias de su consumación y, por tanto, fijar cuándo hay responsabilidad en casos de daño y quién la asume.

A la vista de estas finalidades, y sin olvidar el aspecto de la prohibición y de la represión de las actuaciones generadoras de riesgo que marcan el ámbito de lo lícito, lo cierto es que el contexto de incertidumbre que caracteriza la gestión de los riesgos del progreso nos aboca a un planteamiento jurídico esencialmente precautorio y discrecional, en el que la constatación de la existencia de un riesgo, con independencia de su mayor o menor certidumbre, inclina a adoptar acciones imbricadas a una idea de prevención formulada en sentido negativo: lo que no habría de hacerse o hay que impedir para evitar la consumación de lo que hoy es simple incertidumbre.

\section{El peligro de la tecnocracia y su necesaria conjura}

Los Estados se encuentran desbordados para gestionar las situaciones de riesgo con el grado de rapidez y competencia que éstas demandan. Antes que la Administración, hoy son las grandes empresas y las organizaciones profesionales quienes disponen del mayor y mejor conocimiento científico-técnico. La protección jurídica frente al riesgo aconseja aprovechar estas mejores posibilidades cognitivas del sector privado e inclina a trasladar a éste aspectos esenciales de la determinación del riesgo tolerado, de su valoración y de la toma de decisiones en un entorno de incertidumbre.

El creciente interés jurídico que provoca la actual interpenetración de los respectivos mundos de la técnica y el derecho se justifica sobre todo por el trascendente cambio cualitativo que se ha producido en el establecimiento, verificación y control 
de los requisitos técnicos exigibles a los productos e instalaciones con riesgos ${ }^{4}$. En el terreno de las funciones declarativas y ejecutivas, la complejidad técnica que hoy encierra la gestión del riesgo ha motivado una intensa penetración de la colaboración privada; de tal forma que, en la inmensa mayoría de los casos, son sujetos puramente privados altamente especializados los que ejercitan las funciones de inspección, certificación y autorización de productos y actividades productivas que impliquen un riesgo potencial para la salud, seguridad de las personas o el medioambiente. Junto a ello, la intervención de sujetos privados también se hace presente en el aparentemente más impenetrable ámbito de la función normativa, del establecimiento de la regla de derecho. El mundo de la técnica ha necesitado siempre patrones de comportamiento que se han desarrollado con una marcada autonomía respecto al mundo de las normas jurídicas, a través de un "para-ordenamiento" de la técnica cuya expresión más acabada es el sistema de normalización industrial ${ }^{5}$. El derecho encuentra aquí una fuente plena de conocimiento técnico de la que se sirve para arbitrar soluciones jurídicas en un doble plano: el del conocimiento y valoración jurídica de los riesgos y el de la fijación de los límites del riesgo tolerable. Las normas jurídicas tienden así a centrarse en las cuestiones formales y procedimentales, mientras que la regulación de los aspectos materiales y sustantivos es confiada a "normas técnicas" que surgen de un singular fenómeno de autorregulación privada. Estas "normas técnicas" nacen con voluntad de aplicación voluntaria en el ámbito convencional y contractual privado; sin embargo, el derecho se sirve constantemente de ellas por vía de remisión, confiriendo a dichas normas -inicialmente privadas y voluntarias- fuerza jurídica vinculante.

El recurso al sector privado por la incapacidad e insuficiencia del aparato público determina una modulación de las relaciones Estado-sociedad en el ámbito de la técnica que da entrada a fórmulas de colaboración privada de una intensidad hasta ahora desconocidas, que se articulan mediante la atribución a sujetos privados

\footnotetext{
${ }^{4}$ Dedique a esta cuestión J. A. CARRILIO DONAIRE (2000) El Derecho de la seguridad y calidad industrial, Madrid, Marcial Pons, cuyos planteamientos actualicé en J. A. CarriLo DonaIRE (2009) "Seguridad y calidad productiva: de la intervención policial a la gestión de riesgos": Revista de Administración Pública, 178, 89-142.

${ }^{5}$ Sobre la normalización industrial puede verse mi libro El Derecho de la seguridad... op. cit., 298 y ss; V. Álvarez García (1999) La normalización industrial, Valencia, Tirant lo Blanch; e ID. (1998) "La capacidad normativa de los sujetos privados": Revista Española de Derecho Administrativo, 99, 343-367; R. J. Moles i PLAZA (2001) El régimen jurídico de la normalización técnica, Barcelona, Ariel; S. RodríGuez-CAMPOS GONZÁlez (2002) Calidad industrial e intervención administrativa, Barcelona, Atelier; y M. TARRÉs VIVES (2003) Normas técnicas y ordenamiento jurídico, Valencia, Tirant lo Blanch; e ID. (2003) "Las normas técnicas en el Derecho Administrativo": Documentación Administrativa, 265-266, 151-184.
} 
de un ámbito de capacidad jurídico-pública destinada a satisfacer determinadas exigencias del interés general para cuya consecución gozan de prerrogativas y potestades exorbitantes. Se produce, con ello, una nueva correlación en el ejercicio de poder público entre el Estado y la sociedad en la que la legitimación jurídica del ejercicio de autoridad se fundamenta en el conocimiento experto del que es depositario el sector privado, configurado como un "para-Estado" autorregulado de la técnica.

El mayor problema jurídico que suscita el fenómeno apuntado se centra en la desmaterialización del derecho aplicable, que abandona la determinación sustantiva del umbral de seguridad y del riesgo tolerable, y la disminución de la capacidad decisora de la Administración en el control de su cumplimiento.

El verdadero peligro de que la ciencia y la técnica penetren de este modo en el ámbito del derecho es que se desmorone el sistema asentado de legitimaciones que éste encarna. Nuestra dependencia científico-técnica amenaza seriamente aspectos esenciales de la legitimidad democrática del poder y de la legalidad en la toma de decisiones en el contexto de la prevención y corrección del riesgo. En no pocas ocasiones, las normas jurídicas se ven sustituidas por prescripciones que emanan del mundo de la técnica a las que no cabe concebir ni identificar como decisiones jurídicas en su esencia. Asimismo, la conjura del riesgo mediante la adopción de decisiones en un entorno de incertidumbre, la elección de las concretas medidas y el control de las mismas, depende en buena medida de profesionales y expertos que se sitúan al margen del aparato administrativo y del poder ejecutivo. Con ello, la voluntad normativa y la fuerza directiva del derecho se ve reemplazada por la supuesta legalidad y legitimidad intrínsecas al saber experto. Aquél está necesitado, por tanto, de recuperar para sí la dialéctica de poder y voluntad que respalda y legitima la acción pública en nuestro sistema constitucional.

La atribución de potestades a sujetos privados para el ejercicio de funciones públicas encierra, por tanto, un inmenso peligro, pues los sujetos privados que reciben esas potestades en función de su mejor posicionamiento para afrontar el riesgo son muchas veces los mismos que se lucran generando ese riesgo, y también los que están más expuestos a los intereses del mercado. El recurso a estas fórmulas de colaboración encierra una paradoja difícilmente reductible, pues probablemente - haya situación más peligrosa para la libertad y la seguridad jurídica que un sujeto privado dotado de prerrogativas públicas. Para hacer frente a este peligro hay que evitar el sometimiento del derecho a una intelligentsia científico-técnica que es ajena al sistema de valores que expresa el consenso político y jurídico constitucional, sistema jurídico de valores y postulados que no tienen trasunto en 
el mundo científico, presidido metodológicamente por la racionalidad objetiva y la neutralidad axiológica, cada vez más cuestionada por intereses económicos que orientan y "capturan" la generación del conocimiento desacreditando el argumento de la neutralidad científico-técnica ${ }^{6}$.

Para ello, es preciso diseñar garantías jurídicas de objetividad que compensen la pérdida de dominio público con un mayor protagonismo de los aspectos formales y procedimentales que aseguren la participación de todas las partes interesadas, la imparcialidad y la transparencia en la determinación y valoración del riesgo y en la toma de decisiones para prevenirlo o corregirlo.

En todo caso, la retirada del Estado y las administraciones públicas no debe suponer un menoscabo de los intereses públicos en presencia, sino una mutación del papel que tradicionalmente desempeñaban aquéllas como protagonistas absolutos del eje prevención-corrección del riesgo mediante un sistema objetivo de garantías y mecanismos de supervisión y tutela que permitan una efectiva conducción pública del proceso. De lo contrario correríamos el riesgo de caer en una tecnocracia que hurtaría a las decisiones su fuente de legalidad y de legitimidad.

\section{Labilidad del principio de legalidad en supuestos de riesgos graves y desconocidos: alcance y límites del principio de pre- caución como cláusula habilitadora de medidas excepcionales}

La construcción teórica del Derecho medioambiental ha tenido que enfrentarse de forma recurrente al reconocimiento de poderes implícitos o inmanentes de la Administración que suponen un escollo para el principio de legalidad que debe presidir la adopción de medidas restrictivas de la libertad de empresa. Teorías como la del estado de necesidad, el deber de respetar el orden público o, más recientemente, las obligaciones positivas que derivan de la plena satisfacción de derechos constitucionales como el pleno disfrute del medio ambiente, justifican la atribución al poder ejecutivo un genérico apoderamiento para limitar derechos y libertades mediante medidas que cuentan con un amparo legal más o menos

\footnotetext{
${ }^{6} \mathrm{H}$. JONAS (1995) El principio de responsabilidad. Ensayo de una ética para la civilización tecnológica, Barcelona, Herder; M. González García, J. A. López Cerezo y J. L. Luján López (1996) Ciencia, tecnología y sociedad: una introducción al estudio social de la ciencia y la tecnología, Madrid, Tecnos, 130 ss; E. Agazzı (1996) El bien, el mal y la ciencia. Las dimensiones ética de la empresa científico-técnica, Madrid, Tecnos, 76 ss.; J. ECheverríA (2003) Ciencia y valores, Barcelona, Destino.
} 
evidente 0 , y en esto radica el aspecto más problemático y discutible de estas tesis, que se adoptan al margen de la ley o incluso contra legem en situaciones singulares de necesidad o de excepción.

En el ámbito de la protección medioambiental, estas habilitaciones normativas se enmarcan hoy en el llamado principio de precaución o de cautela emergido del ámbito comunitario europeo y que actualmente plasma el artículo 191 del Tratado de funcionamiento de la Unión Europea en relación con el riesgo medioambiental, aunque las instancias comunitarias han extendido su aplicación a todo el ámbito productivo y de consumo, posibilitando su invocación cuando es urgente intervenir ante un posible peligro para la salud, la seguridad o el medio ambiente, en supuestos en que los datos científicos no permitan una determinación completa del riesgo 7 .

El principio de precaución da cobertura a medidas que se adoptan en situaciones de desconocimiento o de gran incertidumbre científica en las que las autoridades se enfrentan a un delicado dilema: la posibilidad de que una medida restrictiva (por ejemplo, el cierre de una industria o la retirada de un producto del mercado por sus efectos potencialmente dañinos) quede deslegitimada posteriormente si se demuestra científicamente que el riesgo era menor o inexistente, en cuyo caso podrían derivarse acciones de resarcimiento por una reacción jurídica desproporcionada y no justificada; y la hipótesis contraria, que la ausencia de una reacción adecuada frente el riesgo y el posterior daño permita imputar responsabilidad por inactividad o insuficiencia de las medidas en beneficio en este caso de las víctimas.

Por otro lado, la aplicación del principio de precaución no prejuzga el cumplimiento de la legalidad formal por parte de los sujetos generadores del riesgo, que incluso se presupone. Se trata de medidas restrictivas que se adoptan respecto de actividades que pueden cumplir con todas las exigencias legales. No estamos en supuestos de incumplimiento del nivel de seguridad jurídicamente exigible ni ante una prueba plena del carácter "peligroso" de una actividad o producto. La clave de aplicación del principio de precaución es más conjetural e indiciaria: el estado de incerteza y la gravedad del riesgo hipotéticamente asociado a ciertas tecnologías o productos legitiman la adopción de medidas correctivas incisivas,

\footnotetext{
7 J. Esteve Pardo (2003) "Principio de precaución. El Derecho ante la incerteza científica": Revista Jurídica de Cataluña, 102/3, 41-52; M. Rebollo Puig y M. Carrasco Izquierdo (2003) "El principio de precaución y la defensa de los consumidores": Documentación Administrativa, 265-266, 185-236; C. Cierco Selra (2004) "El principio de precaución: reflexiones sobre su contenido y alcance en los Derechos comunitario y español": Revista de Administración Pública, 163, 73-126; e ID. (2005) "El principio de precaución", en J. RUANO GÓMEZ, director (2005) Riesgos colectivos y situaciones de crisis: el desafío de la incertidumbre, Universidad de La Coruña, 17-63.
} 
rescisorias o incluso compulsivas, cuya legitimidad quiere compensarse extremando la proporcionalidad, la temporalidad y la "revisabilidad" de las mismas, así como el rigor en el análisis y valoración del riesgo. Con todo, la cobertura legal que ampara esas medidas es enormemente imprecisa, situándose por debajo del umbral de exigencia que ordinariamente reclaman los principios de legalidad y tipicidad para legitimar la restricción de derechos. Más aún, al tratarse de actividades que formalmente satisfacen el concepto jurídico de seguridad mediante el cumplimiento "normal" de la legalidad, se produce aquí un vaciamiento material del principio de legalidad de una fuerza e intensidad desconocidas para el derecho tradicional.

Las habilitaciones normativas que nuestro ordenamiento consagra para arbitrar medidas de contención de los riesgos especialmente graves o inminentes y de los riesgos desconocidos se conectan claramente al principio de precaución así formulado. La previsión general es que

ante situaciones de riesgo para la salud y seguridad de los consumidores y usuarios, las administraciones públicas competentes podrán adoptar las medidas que resulten necesarias y proporcionadas para la desaparición del riesgo, incluida la intervención directa sobre las cosas y la compulsión directa sobre las personas ${ }^{8}$.

La legislación que desarrolla este principio delimita el presupuesto de la gravedad del riesgo en función de los parámetros de probabilidad, gravedad e inminencia, ya que para calificar un riesgo ha de valorarse conjuntamente la probabilidad de que se produzca un daño y la severidad de éste. Así, se considera riesgo grave aquel que en virtud de tales criterios exija una intervención rápida de la Administración, aun en el caso de que los posibles daños para la salud, la seguridad o el medioambiente no se materialicen inmediatamente. Para atajar estos riesgos posibles o probables se pueden adoptar las medidas con la máxima celeridad o incluso inmediatamente cuando resulten necesarias para garantizar la salud, la seguridad de los consumidores o la preservación del medioambiente. Medidas que, según dispone la legislación, deberán ser congruentes con los motivos que las originen, proporcionadas con los riesgos que afronten $y$, de entre las que reúnan esos requisitos, las menos restrictivas para la libertad. En todo caso, el principio de precaución permite adoptar esas medidas cuando, tras haber evaluado la información disponible, se observe la posibilidad de que haya efectos nocivos, aunque exista incertidumbre científica. En suma, estas habilitaciones fundamentan decisiones de excepción que rompen la regla de derecho ordinariamente aplicable. El principio de precaución se convierte, así, en el derecho "normal" de las

${ }^{8}$ Art. 15 del texto refundido de la Ley de consumidores y usuarios. 
circunstancias excepcionales de riesgo que derivan de la gravedad o inminencia del mismo.

Como no puede ser de otra forma, la excepcionalidad de estas medidas limitativas y su confrontación directa con el principio de legalidad, tipicidad y seguridad jurídica requiere extremar los límites y los rigores de su aplicación. La Comunicación de la Comisión sobre el recurso al principio de precaución, de 2-II-2000, hace referencia a una serie de principios y reglas que ajustan el alcance del principio y condicionan la validez de las medidas adoptadas plasmando límites específicos a la discrecionalidad mucho más afinados que los tradicionales controles basados en el error manifiesto de apreciación, la desviación de poder o la interdicción de la arbitrariedad. Esos límites se refieren, en primer lugar, al presupuesto de hecho para la adopción de estas medidas, siendo esencial que, dada la incertidumbre existente sobre el riesgo advertido, quede acreditada de una forma objetiva y suficiente la prueba de la gravedad e inminencia del mismo. En segundo lugar, en relación con el contenido de las medidas, la clave de aplicación es el principio de proporcionalidad, que obliga a mesurar el alcance de las decisiones en función de la magnitud e inminencia del riesgo, procurando logar un justo equilibrio entre estos factores y el daño a la libertad a través de un adecuado balance costes-beneficios. En tercer lugar, los derechos e intereses afectados requieren la aplicación objetiva y no discriminatoria de las medidas, de modo que guarden conexión y equivalencia con las adoptadas en ámbitos $v$ ocasiones similares. Finalmente, también existen límites específicos que derivan de la naturaleza esencialmente cautelar de las medidas que se fundamentan en el principio de precaución. Si -como antes quedó expuesto- la temporalidad y la reversibilidad son consustanciales a las decisiones de riesgos, en estos casos existe un deber, y no ya una mera posibilidad, de adoptarlas por tiempo limitado y de revisarlas, y en su caso revocarlas, en consonancia con la evolución del conocimiento y la valoración del riesgo.

A mi juicio, el mayor problema jurídico que suscita el fenómeno apuntado consiste en la desmaterialización del derecho aplicable, que abandona en pro del conocimiento técnico la determinación sustantiva del umbral de seguridad y del riesgo tolerable, lo que se corresponde con un mayor apoderamiento de la Administración y con una mayor indeterminación de las medidas que ésta puede adoptar para atajar el riesgo. 